\title{
Study on the Influence of Phase Noise on Communication System
}

\author{
Yang Liu and Ma Ruofei \\ Xi'an Electronic Engineering Research Institute
}

\begin{abstract}
The phase noise plays an important role in the communication system. It is the normalized frequency offset caused by the local oscillator. It not only reduces the signal to noise ratio at the transmitter but also seriously deteriorates the capacity of demodulation at the receiver. In this paper, we analyze the relationship between phase noise and EVM (Error Vector Magnitude), BER (Bit Error Rate), it can provide a theoretical basis for millimeter wave communication system designing.
\end{abstract}

Keywords: Phase Noise, Phase error, EVM, BER.

\section{Introduction}

The single frequency source generated by the local oscillator is used as a reference clock. The synchronization of the entire system depends on the accuracy of this reference source. The communication system performances are also limited by the stability of this reference source. The phase noise is caused by the local oscillator, whereas digital system engineers work with the jitter of the clock. It not only affects the signal quality on the transmitter but also reduces BER on the receiver; therefore, the effects of phase noise should be taken into consideration in communication system designing.

Much research has devoted to several aspects of the study of phase noise on oscillators [1-6]. The theoretical model of phase noise has been analyzed from various viewpoints [1-3]. The constrained capacity of the additive white Gaussian noise channel affected by Wiener phase noise and achievable information rate are investigated in $[4,5]$, which give insights into the design of practical communication system. A review of the main information-theoretic results on channels affected by a time-varying phase noise is studied in [6]. In this paper, we focus on the influence of phase noise on the practical millimetre wave communication system and analyze the relationship between phase noise and EVM (Error Vector Magnitude), BER (Bit Error Rate).

The remainder of the paper is organized as follows. Firstly, we give a general definition of phase noise and a mathematical model to estimate it in section 2. The influence of phase noise at the transmitter and receiver are studied in section 3 . In section 4 , we give some simulations to illustrate the influence of phase noise on communication system and provide practical guidance for communication system design. The summary of this paper is in section 5 .

\section{Definition of Phase Noise}

The SSB (single sideband) phase noise $\theta(t)$ is defined as the ratio of the phase modulation sideband power to the total signal power of the frequency offset signal within the $1 \mathrm{~Hz}$ bandwidth. The SSB phase noise spectrum $L(f)$ can be represented in Eq. (1) and shown in Fig. 1

$$
L(f)=\frac{\text { Phase modulation sideband power }}{\text { Total signal power }}
$$




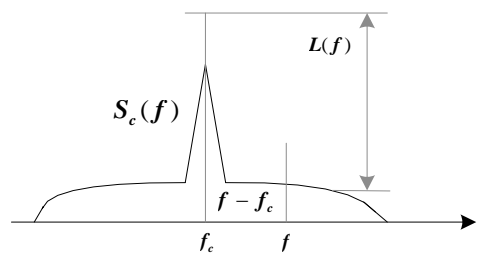

Fig. 1: definition of phase noise spectrum

The output signal with phase noise is given by

$$
C(t)=A \sin \left(2 \pi f_{c}\left(t+\frac{\theta(t)}{2 \pi f_{c}}\right)\right)
$$

Since $\theta(t)<<\frac{\pi}{2}$, Eq. (1) can be rewritten as Eq. (3), where $\omega_{c}=2 \pi f_{c}$ is the angular frequency.

$$
C(t)=A \sin \left(\omega_{c} t\right)+A \theta(t) \cos \left(\omega_{c} t\right)
$$

The DSB (double sideband) power spectrum $S_{c}(f)_{2}$ of the output signal $C(t)$ is given by:

$$
S_{c}(f)_{2}=\frac{P_{s}}{2}\left[\delta\left(f-f_{c}\right)+\delta\left(f+f_{c}\right)\right]+\frac{P_{s}}{2}\left[S_{\theta}\left(f-f_{c}\right)_{2}+S_{\theta}\left(f+f_{c}\right)_{2}\right]
$$

Where $S_{\theta}(f)_{2}$ is the DSB power spectrum of random phase noise $\theta(t), P_{s}$ is the total signal power. $S_{\theta}\left(f-f_{c}\right)_{2}$ and $S_{\theta}\left(f+f_{c}\right)_{2}$ are DSB phase noise power spectral density. The power spectral density $S_{c}(f)$ is represented by

$$
S_{c}(\Delta f)=\frac{1}{2} S_{\theta}(\Delta f)
$$

From Eq. (1), the SSB phase noise spectrum $L(f)$ can be represented by

$$
L(\Delta f)=\frac{S_{c}(\Delta f)}{S_{c}\left(f_{c}\right)}
$$

In the practical communication system, Eq. (6) can be rewritten as

$$
L(\Delta f)=\frac{1}{2} S_{\theta}(\Delta f) \text { in } d B c
$$

Finally, the phase noise can be represented by phase error $\theta_{R M S}^{2}$, it is the mean square of phase jitter .

$$
\theta_{R M S}^{2}=\int_{0}^{\infty} S_{\theta}(f) d f=\int_{0}^{\infty} 2 \times 10^{\frac{L(f)}{10}} d f
$$

\section{Influence on Communication System}

\subsection{Influence on the Transmitter}

A practical structure of the transmitter is shown in Fig. 2. The FPGA is responsible for modulation. The modulated QPSK signal is divided to I/Q stream before accessing to the DA module. The AD module (AD9361) take charge of converting the baseband signal to $1 \mathrm{GHz}$ ( $\max$ is $6 \mathrm{GHz}$ ) and mix with the oscillator signal $\left(f_{0}=32 \mathrm{GHz}\right.$ ) to generate the specific signal. Then, the second and third harmonic components of the output signal $\left(f_{l}=33 \mathrm{GHz}\right)$ are removed by the band-pass filter. Finally, the signal is transmitted via the antenna. 

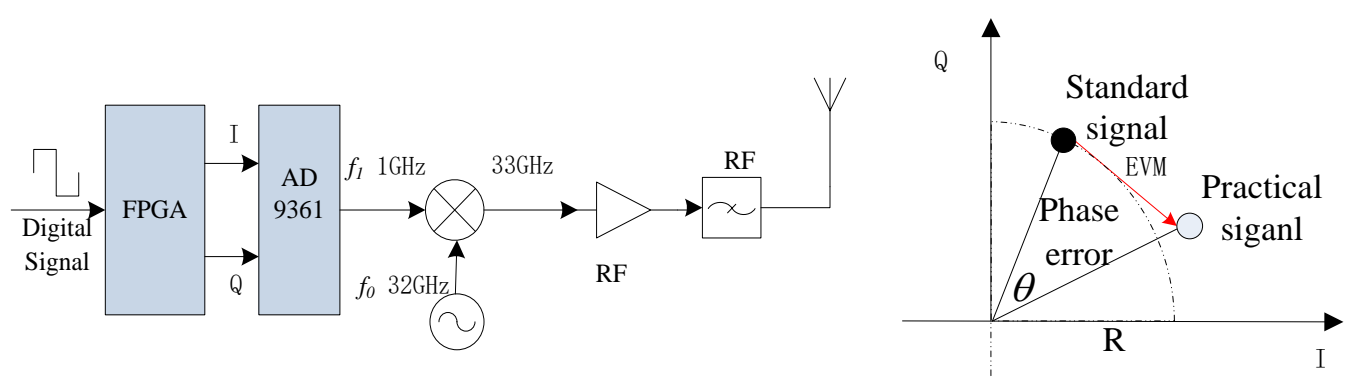

Fig. 2: structure of transmitter

Fig. 3: relationship between phase error and EVM

EVM is a measurement of the difference between the actual waveform and ideal waveform, which determine the precision of modulating at the transmitter. Assume that $Y(k)$ is the sampled complex signal of the transmitter signal during $K T$ time ( $T$ is symbol period), $S(k)$ indicates the ideal signal which is normalized by the unit circle.

$$
Y(k)=\left[C_{0}+C_{1}(S(k)+E(k))\right] W^{k}
$$

where $W=e^{\Delta r+j \Delta a}$ represents the rate of change of amplitude $\Delta r$ and frequency offset $\Delta a . C_{0}$ means the unbalanced of the quadrature modulator. $C_{1}$ is the output signal power and phase at the transmitter, $E(k)$ is the error vector, where $k=1,2, \ldots, N$ is the $k t h$ sample of error vector.

$$
E V M_{R M S}=\left[\frac{1}{N} \sum_{k=1}^{N}|E(k)|^{2}\right]^{\frac{1}{2}}
$$

The error vector can be estimated by the phase error of the local oscillator as shown in Fig. 3, $\mid \overrightarrow{|E|} \approx R \cdot \theta$. Where $R$ is the unit circle radius. Thus the relationship of phase error and EVM can be represented by

$$
\begin{aligned}
E V M_{R M S} & =\left|E V M_{R M S}\right| / R \\
& \approx 100 \% R \bullet \theta_{R M S} / R=100 \% \bullet \theta_{R M S}
\end{aligned}
$$

EVM is a performance index of transmitter and usually used to measure the quality of the transmitted signal in military communications. Suppose $C_{0}=0$ and the output signal power normalized as $C_{1}=1$, the relationship between EVM and SNR at the transmitter can be simply given by

$$
E V M_{R M S}=\left[\frac{\frac{1}{N} \sum_{k=1}^{N}|Y(k)-S(k)|^{2}}{\frac{1}{N} \sum_{k=1}^{N}|S(k)|^{2}}\right]^{\frac{1}{2}} \approx\left[\frac{\frac{1}{N} \sum_{k=1}^{N}|E(k)|^{2}}{\frac{1}{N} \sum_{k=1}^{N}|S(k)|^{2}}\right]^{\frac{1}{2}}=\left[\frac{1}{S N R}\right]^{\frac{1}{2}}
$$

There is an inverse relationship between SNR and the square of EVM. From Eq. (12), we find that the phase noise can directly decided the system capacity based on SNR.

\subsection{Influence on the Receiver}

A super-heterodyne receiver is employed at the receiver. The RF signal from the receiving antenna is magnified by LNA (low noise amplifier) and changed to the intermediate frequency signal by mixing with the signal generated by local oscillator. After A/D sampling and data processing, the RF signal is changed to the baseband signal. The coherent demodulation is employed at the receiver. The received signal $C(t)$ with phase noise caused by local oscillator at the receiver is represented by

$$
C(t)=\cos \left(2 \pi f_{0} t+\theta(t)\right)
$$

where $f_{0}$ is the carrier frequency, $\theta(t)$ is the phase noise at receiver. Suppose the phase noise at receiver follow the Gaussian noise model, the power spectrum of phase noise at receiver is

$$
\rho(\theta)=\frac{1}{\sqrt{2 \pi \sigma^{2}}} e^{-\frac{\theta^{2}}{2 \sigma^{2}}}
$$

The BER with phase noise at receiver is given by 


$$
p_{e}=\int_{-\pi}^{\pi} \rho(\theta) p_{e}\{e \mid \theta(k)=\theta\} d \theta
$$

When the coherent demodulation for QPSK modulation is applied, the bit error probability for a given value of $\theta$ can be given by Eq. (16), where SNR is the signal to noise ratio at receiver [7].

$$
\begin{aligned}
P_{e-Q p s k}(e \mid \theta(k)=\theta)= & \frac{1}{2} \operatorname{erfc}(\sqrt{S N R}(\cos \theta+\sin \theta)) \\
& +\frac{1}{2} \operatorname{erfc}(\sqrt{S N R}(\cos \theta-\sin \theta))
\end{aligned}
$$

Combining Eq. (11), (12), (15) and (16), the relationship between phase noise and BER can be represented by

$$
p_{e}=\frac{1}{2} \int_{-\pi}^{\pi} \rho(\theta)\left(\operatorname{erfc}\left(\frac{\cos \theta+\sin \theta}{\theta}\right)+\operatorname{erfc}\left(\frac{\cos \theta-\sin \theta}{\theta}\right)\right) d \theta
$$

\section{Simulation}

In a practical communication system, we get a single sideband power spectrum of phase noise (see Fig. 4.) at the transmitter.

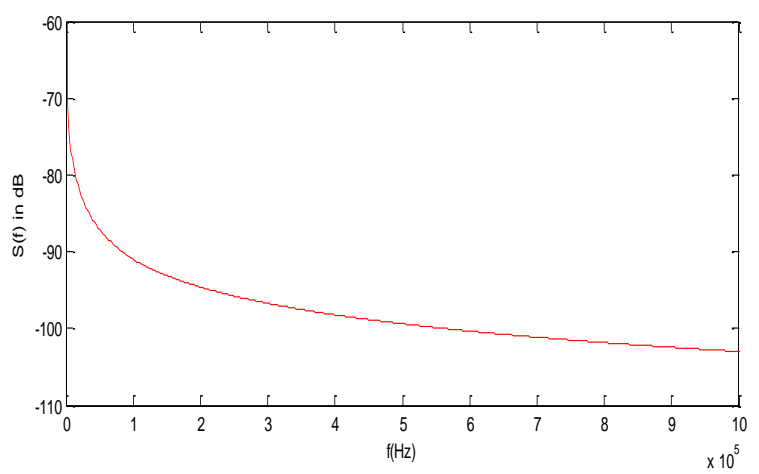

Fig. 4: single sideband power spectrum of phase noise at transmitter

We can get the random phase jitter value by taking angle as a unit from Eq. (8),

$$
\theta(t)=\frac{180}{\pi} \sqrt{\int_{0}^{\infty} 2 \times 10^{\frac{L(f)}{10}} d f}
$$

The phase error is calculated by frequency offset subsection integral shown in Table. 1.

Table.1. Phase error values for different frequency offset subsections

\begin{tabular}{|c|c|c|c|c|}
\hline Subsection & $\begin{array}{c}\text { frequency offset } \\
f_{l}(\mathrm{~Hz})\end{array}$ & $\begin{array}{c}\text { frequency offset } \\
f_{2}(\mathrm{~Hz})\end{array}$ & $\begin{array}{c}\text { Phase noise } \\
(\mathrm{dBc} / \mathrm{Hz})\end{array}$ & Phase error \\
\hline 1 & 100 & $1 \mathrm{~K}$ & $-48 \sim-60$ & $7.02^{\circ}$ \\
\hline 2 & $1 \mathrm{~K}$ & $10 \mathrm{~K}$ & $-60 \sim-68$ & $5.90^{\circ}$ \\
\hline 3 & $10 \mathrm{~K}$ & $100 \mathrm{~K}$ & $-68 \sim-78$ & $4.61^{\circ}$ \\
\hline 4 & $100 \mathrm{~K}$ & $1 \mathrm{M}$ & $-78 \sim-92$ & $2.4^{\circ}$ \\
\hline Average result & \multicolumn{5}{|c|}{$4.98^{\circ}$} \\
\hline
\end{tabular}

The phase error is about $4.98^{\circ}$ in $1 \mathrm{MHz}$. We can get $E V M_{R M S}=8.7 \%$ by Eq. (11) and SNR=21dB。It means that the SNR of output signals at transmitter only can reach $21 \mathrm{~dB}$ by the power spectrum of phase noise given in Fig. 4. It is important for system designing in term of capacity, transmission rate and so on.

Then, we take QPSK communication system as an example to illustrate the phase error influence on demodulation at receiver. The phase error is set as $2^{\circ}, 5^{\circ}$ and $7^{\circ}$ in the simulation. 


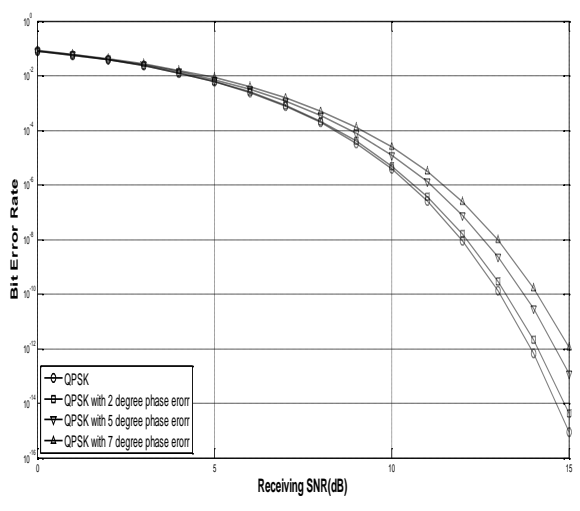

Fig. 5: BER performance in AWGN channel

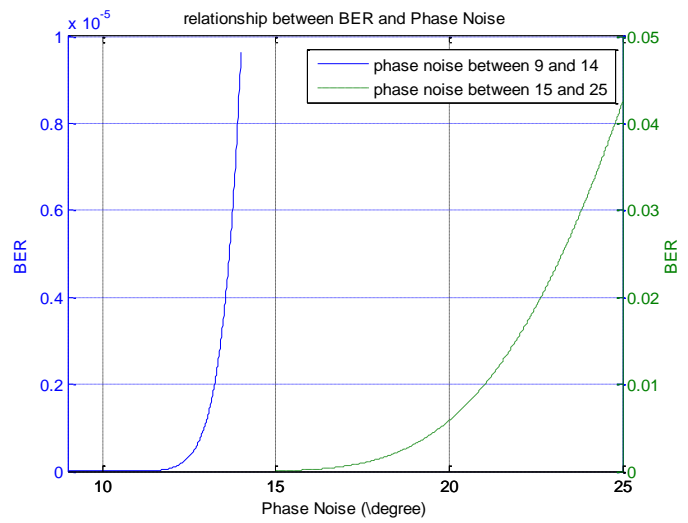

Fig .6: Relationship between phase noise and BER

As shown in Fig. 5, the simulation result indicates that the BER is worse when the phase error increases. When the phase error is $5^{\circ}$, the capability of demodulation at receiver degenerates about $0.6 \mathrm{~dB}$ compared to theoretical value when the BER threshold is $10^{-5}$. When the phase error is $7^{\circ}$, the capability of demodulation at receiver degenerates about $1 \mathrm{~dB}$ compared to theoretical value when the BER threshold is $10^{-10}$. In Fig. 6 , the simulation result indicates that the relationship between phase noise and BER without considering channel noise. The BER almost increase linearly with the phase noise, when it is more than $20^{\circ}$. When the phase noise is less than $10^{\circ}$, the BER is almost equal to 0 .

\section{Conclusion}

The phase noise caused by local oscillator plays an important role in the millimetre wave communication system. In this paper, we systematically analyse the effects of phase noise at the transmitter and receiver. We get the relationship between phase noise and EVM, BER. It can provide a theoretical basis for millimetre wave communication system designing. Generally, the relationship between phase noise and BER also can be applied to other modulation.

\section{References}

[1] F pepe, David B. " A General Theory of Phase Noise in Trans conductor-Based Harmonic Oscillators." IEEE Transactions on Circuits and Systems I: Regular Papers 64.2 (2017): 432-445.

[2] F. L. Traversa, M. Bonnin, F. Corinto, F. Bonani. "Noise in oscillators: a review of state space decomposition approaches. Journal of Computational Electronics , 14.1(2015):51-61.

[3] X Huang, W Fu, P Chen, H Huang. "Physical model of phase noise in feedback oscillator".2013 Joint European Frequency and Time Forum \& International Frequency Control Symposium (EFTF/IFC). (2013):952955.

[4] UL Rohde, AK Poddar, "Phase noise measurement techniques, associated uncertainty, and limitations." 2013 Joint European Frequency and Time Forum \& International Frequency Control Symposium (EFTF/IFC). (2013):438-441.

[5] Goebel, Bernhard, et al. "Calculation of mutual information for partially coherent Gaussian channels with applications to fiber optics." IEEE Transactions on Information Theory 57.9 (2011): 5720-5736.

[6] Colavolpe, Giulio. "Communications over phase noise channels: a tutorial review." International Journal of Satellite Communications and Networking 32.3 (2014): 167-185.

[7] Shafik, Rishad Ahmed, Md Shahriar Rahman, and AHM Razibul Islam. "On the extended relationships among EVM, BER and SNR as performance metrics." Electrical and Computer Engineering, 2006. ICECE'06. International Conference on. IEEE, 2006. 